\title{
On Superposition by the Aid of Dissection.
}

\author{
By R. F. Mulrhead, M.A., B.Sc.
}

What do we mean when we assert that one plane figure is equal to another? In trying to find a satisfactory answer to this question I was led to consider the subjects treated in this paper.

Euclid's Axiom: Magnitudes which coincide are equal, may be taken as defining the nature of geometrical equality: but it obviously does not apply to any but congruent figures. We must therefore have a more comprehensive definition. I suppose the conception tacitly used by mathematicians is that magnitudes are equal which can be so dissected that for each part of one there is a corresponding part of the other which is congruent to it. This would be sufficient for elementary Geometry, while for higher Geometry the method of limits would be needed in addition, and magnitudes would have to be recognised as equal if pairs of mutually congruent parts could be subtracted from them continually until the remainders were both infinitesimal.

Now the first proposition of Euclid in which equal non-congruent figures occur, is the 3ōth of Book I. But there it is to be noted that the two parallelograms are proved to be equal, not directly by dissecting them into mutually congruent parts, but by the aid of the axiom that if equals be taken from equals, the remainders are equal. However, a very simple method of dissection rendering superposition possible in this case is well known, and is given in some school editions of Euclid.

The more general question arises, as to whether any two equal rectilinear plane figures can be rendered superposable by dissection into a finite number of parts. I shall show that this question may be answered in the affirmative.

Prop. 1. Any two rectilinear figures, or systems of rectilinear figures, $A$ and $B$, can be dissected into pairs of equal triangles, one triangle of each pair belonging to $A$ and the other to $B$.

By joining vertices, we can divide both $A$ and $B$ into triangular areas. Suppose the system $\mathrm{A}$ gives $m$, and the system $\mathrm{B}, n$ triangles. Consider any pair of triangles, one from $A$ and the other from $B$. If they are equal, cut off both, and $A$ is left with $m-1, B$ with $n-1$ triangles. But if the triangle in $A$ is greater than that in $B$,

$8 \mathrm{Vol}, 14$ 
cut off from the former a part equal to the latter, and cut off the latter, and $\mathrm{A}$ is left with $m, \mathrm{~B}$ with $n-1$ triangles. Thus the total number of triangles is diminished by two or one according as the triangles chosen are equal or unequal. By repeating this process, we dissect $A$ and $B$ as required, and the number of pairs of congruent triangles cannot exceed $m+n$.

Prop. 2. Any two equal triangles can be made superposable by dissection.

Case 1. If the triangles have two sides of one equal to two sides of the other, and the contained angles supplementary, they may be placed as the triangles ABC, DBC in Fig. 40. Then by joining the mid points of $\mathrm{AB}$ and $\mathrm{BD}$ to $\mathrm{C}$ we divide the triangles into two pairs of congruent triangles marked 1,1 and 2,2 .

Case 2. If the triangles have a side of one equal to a side of the other, let them be placed with this as common base, but on opposite sides of it as in Fig. 41, where ABC, DBC are the equal triangles.

Join $\mathrm{AD}$ which will be bisected by $\mathrm{BC}$ in $\mathrm{E}$. Then $\mathrm{ABE}, \mathrm{DBE}$ are triangles related as in Case 1, and can therefore be dissected as required. So also the triangles AEC, DEC. Thus we have four pairs of congruent triangles 1,$1 ; 2,2 ; 3,3 ;$ and 4,4 .

But this simple construction fails when $A D$ falls without the given triangles. In such a case, as in Fig. 42 , where the line AD cuts $\mathrm{BC}$ produced in $\mathrm{E}$, a further construction is necessary.

$A$ s before, join $\mathrm{E}$ to the mid points of $\mathrm{AB}$ and $\mathrm{AC}$. Then take points $\mathrm{F}, \mathrm{G}, \mathrm{H}, \mathrm{K}$, etc., such that $\mathrm{EF}=\mathrm{FG}=\mathrm{EH}=\mathbf{H K}$, etc., each $=\mathrm{BC}$, and through these points draw lines parallel to the lines previously drawn from $\mathbf{E}$. Let a similar construction be made for the lower triangle $\mathrm{BCD}$, and we get $\mathrm{ABC}$ and $\mathrm{DBC}$ dissected as required, the pairs of congruent parts being indicated as before by numerals. (Observe the different order of the parts in $A B C$ and BCD.)

It is obvious that here also the number of parts will be finite.

Case 3. If the triangles have no pair of equal sides, but are equal in area, let us construct an intermediate triangle equal to either of the given triangles in area, and having one side common to each of them. This is obviously always possible. Let the given triangles be called $P$ and $Q$, and the intermediate one, $R$. As in Case 2, let $P$ and $R$ be dissected into mutually congruent parts, and also $Q$ and $R$. Then $R$ is dissected by two sets of lines. Let each 
part of $P$ be then dissected in the same manner as the corresponding part of $R$ is dissected by the lines of the second part of the construction, and let the parts of $Q$ be similarly treated. Then $P$ and $Q$ are obviously dissected as required.

Prop. 3. (Corollary to the foregoing). Any two plane rectilinear figures of equal area can be made superposable by dissection.

I made some attempts to carry out similar dissections for solid figures bounded by plane faces, but though I succeeded in the case of parallelepipeds and prisms, I failed in the case of tetrahedra and other pyramids. It is to be remarked that precisely at this point in the theory of solids does Euclid discontinue the use of the elementary methods of Book XI., relegating the theory of pyramids (with that of circular areas, etc.) to Book XII. where a method of Exhaustions is used.

It seems to me probable for certain reasons that where I failed, the problem is insoluble, i.e., that in general it is impossible to render two tetrahedra of equal volume congruent by means of dissection into a finite number of parts, and $I$ am not sure but that this impossibility may be conclusively demonstrable. So far, however, I have not arrived at a satisfactory demonstration.

Probably, also, there is a connection between the "superposability" of two solids of equal volume or of two plane figures of equal area and the possibility of proving their equality by elementary geometrical methods, such that one involves the other.

By "superposability" I here mean capability of dissection into a finite number of pairs of congruent parts, one from each figure.

To prove this connection, it would be necessary to show that all the axioms relating to geometrical equality hold good also with regard to "superposability," using this word in the sense just explained. For example we should have to show that if "superposables" be added to "superposables," the wholes are "superposable," etc. I find that the only axiom which presents any difficulty is that corresponding to Euclid's Axiom 3, viz: if "superposables" be taken from "superposables" the remainders are "superposable," which again can be made to depend on this : if congruents be taken from congruents the remainders are "superposable." I have not yet succeeded in establishing this generally, but I find that a construction, of which Fig. 42 is a particular case, goes a good way towards 
it: in fact it demonstrates the axiom for solids with the exception of a special class of cases. But into these questions I shall not enter further at present.

After I had obtained practically all the results given in this paper, $I$ found that a good deal had been already done by others, though in a somewhat different way. In particular, the general problem of Prop. 3 above is completely solved in a paper by Robert Brodie, published in the T.R.S.E., Vol, XXXVI. part 2, p. 307, entitled "On Professor Kelland's Problem on Superposition," in which he refers to some previous papers by Kelland in earlier volumes. Again, Perigal (Messenger of Mathematics, II. p. 103) gives a solution for the case of Euclid I. 47, which is generalised to some extent by Harry Hart (Messenger, VI. p. 150).

\section{On the Flementary Differentiations.}

By R. F. Muirhead, M.A., B.Sc. 